\title{
ESTUDO SOBRE A OCORRÊNCIA DE ACIDENTES DE TRABALHO COM MATERIAL BIOLÓGICO EM PROFISSIONAIS DE ENFERMAGEM DE UM HOSPITAL
}

\author{
Beatriz Amsberg Calazans de Oliveira ${ }^{1}$, Ana Cláudia Garabeli Cavalli Kluthcovsky², Fábio Aragão Kluthcovsky³
}

RESUMO: Este estudo objetivou verificar a ocorrência de acidentes de trabalho com material biológico em técnicos e auxiliares de enfermagem que atuam em hospital. Participaram 81 funcionários, que informaram sobre dados sociodemográficos, do trabalho e sobre acidentes com material biológico nos últimos 2 anos. Dos trabalhadores pesquisados, $17,3 \%$ sofreram acidentes. Comparando-se os trabalhadores que sofreram acidente com os que não sofreram, a única variável estatisticamente significativa foi o tempo de trabalho na instituição $(\mathrm{p}=0,04)$, com associação entre tempo de serviço menor de 2 anos e ocorrência de acidente. Todos os 16 acidentes relatados foram do tipo percutâneo, a maioria envolveu sangue e dedos das mãos, a agulha foi o principal agente causador, ocorreu durante o descarte de material, e a maioria dos funcionários não utilizava equipamento de proteção individual. O profissional de enfermagem deve ser orientado sobre os riscos no trabalho e a importância da biossegurança

PALAVRAS-CHAVE: Enfermagem; Exposição a agentes biológicos; Acidentes de trabalho; Saúde do trabalhador; Hospitais.

\section{STUDY OF WORK-RELATED ACCIDENTS WITH BIOLOGICAL MATERIALAMONG NURSING PROFESSIONALS AT A HOSPITAL}

\begin{abstract}
This study objectified to verify the occurrence of work-related accidents with biological material involving hospital nursing auxiliaries and technicians. Eighty one employees participated, providing socio-demographic work data and biological material-related accidents within the last two years. From the researched workers, $17.3 \%$ had suffered accidents. By comparing the workers who have not suffered accidents with the ones who have, the only statistically significant variable $(p=0.04)$ was the employment time in the institution $(p=0.04)$, associated with less than 2 years of employment time and the occurrence of accidents. Concerning the 16 accidents mentioned, all of them were percutaneous, most of them involving blood and fingers, having the needle as the main responsible during material disposal and most employees were not using personal protection equipment. The nursing professional must be oriented about work risks and the importance of biosafety.
\end{abstract}

KEYWORDS: Nursing; Exposure to biological agents; Accidents occupational; Occupational health; Hospitals

\section{ESTUDIO SOBRE LA OCURRENCIA DE ACCIDENTES DE TRABAJO CON MATERIAL BIOLÓGICO CON PROFESIONALES DE ENFERMERÍA DE UN HOSPITAL}

\begin{abstract}
RESUMEN: Este estudio tuvo como objetivo verificar la ocurrencia de accidentes de trabajo con material biológico en técnicos y auxiliares de enfermería que actúan en hospital. Participaron 81 funcionarios, que informaron sobre datos sociodemográficos, del trabajo y acerca de accidentes con material biológicos en los últimos dos años. De los trabajadores entrevistados, 17,3\% sufrieron accidentes. Comparándose los trabajadores que no sufrieron accidente con aquellos que lo sufrieron, la única variable estadísticamente significativa fue el tiempo de trabajo en la institución ( $\mathrm{p}=0,04)$, con asociación entre el tiempo de servicio menor de dos años y la ocurrencia de accidentes. De los 16 accidentes mencionados, todos fueron del tipo percutáneo; la mayoría envolvió la sangre y los dedos de la mano; la aguja fue el principal agente causador: ocurrió durante el descarte del material, la mayoría de los funcionarios no utilizaba equipamientos de protección individual. El profesional en el área de enfermería debe ser orientado acerca de los riesgos en el trabajo y sobre la importancia de la bioseguridad
\end{abstract}

PALABRAS CLAVE: Exposición a agentes biológicos; Accidentes de trabajo; Salud laboral.

\footnotetext{
${ }^{1}$ Aluna do Curso de Graduação em Enfermagem da Universidade Estadual do Centro-Oeste-UNICENTRO.

${ }^{2}$ Médica. Mestre em Enfermagem em Saúde Pública. Professora Assistente da UNICENTRO. Departamento de Enfermagem.

${ }^{3}$ Médico. Mestre em Enfermagem em Saúde Pública. Professor Assistente da UNICENTRO. Departamento de Enfermagem.
}

Autor Correspondente:

Beatriz Amsberg Calazans de Oliveira

Rua Pedro Siqueira, 659 - 85070-190 - Guarapuava-PR.

Recebido: 04/12/07

E-mail: beatriz-cal@hotmail.com

Aprovado:12/05/08

Cogitare Enferm 2008 Jan/Mar; 13(2):194-205 


\section{INTRODUÇÃO}

Entre os trabalhadores da área da saúde, os profissionais de enfermagem constituem uma categoria de indivíduos expostos a riscos variados, pois permanecem por mais tempo e em contato direto com os pacientes, em virtude da rotina profissional ${ }^{(1)}$. Os riscos ocupacionais, causados por fatores químicos, físicos, mecânicos, biológicos, ergonômicos e psicossociais, podem ocasionar doenças e acidentes de trabalho ${ }^{(2)}$.

O hospital é um local que dispõe de serviços específicos à população e nele estão uma variedade de ações relacionadas à saúde que podem expor seus funcionários à uma ou várias cargas como a exposição às doenças infecto-contagiosas ${ }^{(3)}$. Os profissionais ficam expostos a acidentes com materiais biológicos humanos (sangue, secreções e excreções), podendo levar a doenças profissionais aguda, crônica ou até mesmo à morte ${ }^{(4)}$. Alguns estudos realizados com trabalhadores da saúde demonstraram que o grupo com maior percentual de acidentes de trabalho com material biológico foi o dos profissionais de enfermagem ${ }^{(5-10)}$.

Os acidentes com material biológico são um risco de transmissão do HIV, sendo este risco de 0,3\% em acidentes percutâneos, 0,09\% após contato com mucosa, e ainda não precisamente quantificado, quando da exposição à pele não-íntegra. $\mathrm{O}$ vírus da hepatite $C$ possui uma incidência média de soroconversão após exposição percutânea com sangue comprovadamente infectado por ele de $1,8 \%$, podendo sofrer uma variação de 0 a $7 \%$. Em acidente percutâneo com sangue infectado pelo vírus da hepatite B e com presença de HBeAg (antígeno "e" do vírus da Hepatite B), o risco da hepatite clínica varia de 22 a 31\%. Quando o paciente-fonte apresenta apenas o HBsAg (antígeno "s" do vírus da hepatite B - HbeAg negativo), o risco de hepatite clínica varia de 1 a $6 \%$ e o de soro conversão de 23 a $37 \%{ }^{(11)}$

Estudos têm sido realizados sobre os diversos aspectos de acidentes de trabalho com material biológico em profissionais da saúde no Brasil e em outros países ${ }^{(5-}$ $10,12-15)$, e especialmente na equipe de enfermagem ${ }^{(16-}$ ${ }^{21)}$. Contudo, não é possível conhecer, no Brasil, a magnitude do problema relacionado a acidentes de trabalho com material biológico, pois há escassez de dados sistematizados sobre esses acidentes ${ }^{(2)}$.

Assim, este estudo justifica-se, pois os dados obtidos podem subsidiar análise sobre a ocorrência desses acidentes e indicar intervenções específicas para minimizar os danos e melhorar as condições de trabalho. Além disso, entende-se a importância do tema, pois acidentes com material biológico em profissionais de enfermagem têm repercussões biopsicossociais para o trabalhador, sua família e para a sociedade em geral, pelos encargos gerados à previdência e ao sistema de saúde e para a instuituição em que trabalha.

Este estudo teve como objetivos verificar a ocorrência de acidentes de trabalho com material biológico em técnicos e auxiliares de enfermagem, nos últimos dois anos trabalhados, em um hospital geral no interior do Estado do Paraná, caracterizar os profissionais quanto aos dados sociodemográficos e do trabalho e descrever os acidentes de trabalho com material biológico ocorridos e verificar as condutas pós-acidente.

\section{MÉTODOS}

Trata-se de estudo descritivo, transversal, de abordagem quantitativa ${ }^{(22)}$. A pesquisa foi realizada em um hospital localizado em um município do interior do Paraná. Esse hospital é referência para uma região com aproximadamente 500 mil habitantes. Ainstituição possui 154 leitos, sendo 121 cadastrados para atendimento ao Sistema Único de Saúde (SUS), divididos em Clínicas Médica, Cirúrgica, Pediátrica, Neurológica, Psiquiátrica e Obstétrica, além de serviço de Pronto Socorro 24 horas, Unidade de Terapia Intensiva (UTI) Neonatal e Geral e Unidade Coronariana. Conta com serviços de Quimioterapia e Estudo Hemodinâmico. É referência em atendimento ao trauma, gestação de alto risco e alta complexidade em neurocirurgia, atendimento oncológico e cirurgia cardíaca.

Para a realização da pesquisa foi solicitada autorização da instituição hospitalar e o estudo foi aprovado pelo Comitê de Ética em Pesquisa, OF.COMEP-0172/2006, protocolado sob número 136/ 2006. Antecedendo a coleta de dados, todos os sujeitos da pesquisa foram esclarecidos sobre o objetivo do estudo, modo de aplicação do questionário e tratamento dos dados, que a participação seria voluntária, os resultados seriam tratados com confidencialidade e foi garantido o anonimato das informações. Também assinaram o Termo de Consentimento Livre e Esclarecido, nos termos da Resolução 196/96 do Conselho Nacional de Ética em Pesquisa.

O instrumento utilizado para a coleta de dados foi um questionário contendo questões objetivas ${ }^{(22)}$, préelaboradas cuja construção baseou-se na literatura( ${ }^{(7,18)}$. Esse questionário foi submetido a três juízes para 
validaçãoaparente e de conteúdo, todos peritos na temática e na metodologia proposta, sendo duas enfermeiras e uma médica. Foram realizados os ajustes necessários, atendendo às sugestões dos juízes. Os dados investigados incluíram a caracterização dos sujeitos da pesquisa quanto aos dados sociodemográficos, do trabalho, ocorrência do acidente de trabalho, estado vacinal, caracterização dos acidentes ocorridos e procedimentos pós-acidentes.

Foram considerados neste estudo os acidentes com material biológico ocorridos na instituição, sendo que outros tipos de acidentes de trabalho, ou aqueles ocorridos em outros empregos, não foram considerados. Os acidentes de trabalho com material biológico considerados na pesquisa foram: exposição em pele nãoíntegra, mucosa e acidente percutâneo, assim definidos $^{(11)}$ : Exposição cutânea (pele não-íntegra), por exemplo, contato com pele com dermatites ou feridas abertas; Exposição em mucosa, por exemplo, quando há respingo na face envolvendo olhos, nariz, boca ou genitália; Exposição percutânea (lesões perfurantes ou cortantes), por exemplo, agulhas, bisturi, vidrarias.

Os critérios de inclusão no estudo foram: ser profissional da enfermagem (técnico em enfermagem ou auxiliar em enfermagem) e fazer parte do quadro funcional da instituição. Os critérios de exclusão foram: ser estagiário; estar afastado das atividades profissionais por qualquer motivo durante o tempo de coleta dos dados; ser profissional de enfermagem de nível superior ou recusar a participar do estudo.

Foi realizado um estudo-piloto com profissionais de enfermagem do hospital (técnicos e auxiliares em enfermagem) onde foi realizada a pesquisa, para verificar o entendimento do questionário pelos pesquisados. Como não foram necessárias modificações no questionário, os resultados do estudo piloto foram incluídos na pesquisa.

No período de coleta de dados, o hospital contava com 113 profissionais de enfermagem (técnicos e auxiliares de enfermagem), sendo que 9 estavam afastados de suas atividades. Assim, de um total de 104 profissionais, $81(77,9 \%)$ participaram do estudo e houve $23(22,1 \%)$ recusas. Os profissionais que se recusaram a participar referiram estar muito ocupados ou sem interesse em participar. A coleta de dados ocorreu de outubro a dezembro de 2006, sendo que o questionário para coleta de dados foi aplicado individualmente para cada profissional de enfermagem, no hospital e em horário pré-estabelecido.

Os dados foram processados em microcomputador, no banco de dados tipo Excel, com dupla digitação. O tratamento dos dados obtidos foi realizado por meio de análise das freqüências absoluta e relativa, e medidas estatísticas descritivas. Histogramas e teste de Kolmogorov-Smirnov foi usado para checar normalidade das variáveis. Foram utilizados os testes Exato de Fisher para comparar proporções e o teste de Mann Whitney para comparar medianas. Um valor de $\mathrm{p}<0,05$ foi considerado para indicar significância estatística ${ }^{(24)}$.

\section{RESULTADOS E DISCUSSÃO}

A Tabela 1 apresenta a caracterização dos profissionais de enfermagem, segundo variáveis sociodemográficas, do trabalho, ocorrência de acidente de trabalho com material biológico e estado vacinal contra hepatite B. Constata-se que 66 entrevistados (81,5\%) eram mulheres. A predominância de mulheres também foi encontrada por outros pesquisadores em estudos sobre acidentes de trabalho com profissionais de enfermagem ${ }^{(8,13,16,18,23)}$ e profissionais da saúde ${ }^{(7,9-}$ 10,12). A predominância do sexo feminino na enfermagem tem uma relação histórica, pois até o final da Idade Média o cuidar dos pacientes era realizado por mulheres religiosas, virgens, viúvas e nobres, com a finalidade de praticar a caridade ${ }^{(23)}$.

A idade variou de 20 a 56 anos, com média de 35,5 anos $(\mathrm{DP} \pm 9,4)$. Quanto ao estado civil, 44 profissionais (54,3\%) tinham companheiro (casado ou vivendo maritalmente) e 37 (45,7\%) não tinham companheiro (viúvo, solteiro ou separado). Resultados semelhantes foram encontrados em outros estudos com profissionais de enfermagem quanto à idade ${ }^{(16,23)}$ e ao estado civil ${ }^{(13)}$.

Houve predominância da categoria profissional de técnicos de enfermagem com 58 sujeitos (71,6\%), em relação aos auxiliares de enfermagem com 23 sujeitos (28,4\%). Quanto ao tempo de trabalho na instituição foi encontrado uma média de 6,7 anos (DP $\pm 6,9)$, com limites de 5 dias a 32 anos, ou seja, profissionais recém-contratados e profissionais que trabalhavam na instituição há muitos anos.

Levando em consideração a jornada de trabalho, 41 profissionais $(51,3 \%)$ trabalhavam de 6 a 8 horas por dia e 39 (48,7\%) trabalhavam 12 horas. Quanto ao turno de trabalho, 43 sujeitos da pesquisa (53,7\%) trabalhavam no turno diurno.

A maioria dos trabalhadores, 45(55,5\%), revelou não ter outra atividade remunerada e 36 (44,5\%) possuía 
Tabela 1 - Distribuição dos profissionais de enfermagem quanto as variáveis sociodemográficas, do trabalho, acidentes com material biológico e estado vacinal. Paraná, set 2004-dez 2006 (n=81)

\begin{tabular}{|c|c|c|c|c|}
\hline Categorias & Variáveis & $\mathbf{n}$ & $\%$ & Média (DP) \\
\hline \multirow[t]{7}{*}{ Sociodemográficas } & Sexo & & & \\
\hline & Feminino & 66 & 81,5 & \\
\hline & Masculino & 15 & 18,5 & \\
\hline & Idade em anos & & & $35,5(9,4)$ \\
\hline & Estado Civil & & & \\
\hline & Com companheiro(a) & 44 & 54,3 & \\
\hline & Sem companheiro(a) & 37 & 45,7 & \\
\hline \multirow[t]{13}{*}{ Trabalho } & Categoria Profissional & & & \\
\hline & Técnico de enfermagem & 58 & 71,6 & \\
\hline & Auxiliar de enfermagem & 23 & 28,4 & \\
\hline & Tempo de trabalho na instituição em anos & & & $6,7(6,9)$ \\
\hline & Jornada de trabalho* & & & \\
\hline & 6-8 horas & 41 & 51,3 & \\
\hline & 12 horas & 39 & 48,7 & \\
\hline & Turno de trabalho* & & & \\
\hline & Diurno & 43 & 53,7 & \\
\hline & Noturno & 37 & 46,3 & \\
\hline & Outra atividade remunerada & & & \\
\hline & Não & 45 & 55,5 & \\
\hline & Sim & 36 & 44,5 & \\
\hline \multirow{3}{*}{$\begin{array}{c}\text { Acidentes de } \\
\text { Trabalho }\end{array}$} & Acidentes com material biológico nos últimos 2 anos $\dagger$ & & & \\
\hline & Não & 67 & 82,7 & \\
\hline & Sim & 14 & 17,3 & \\
\hline \multirow[t]{5}{*}{ Estado vacinal } & Imunização contra Hepatite B & & & \\
\hline & Imunização completa & 67 & 82,7 & \\
\hline & Imunização incompleta & 11 & 13,5 & \\
\hline & Sem imunização & 2 & 2,5 & \\
\hline & Não informado & 1 & 1,3 & \\
\hline
\end{tabular}

* Um funcionário folguista foi excluído

† Considerando exposição em pele não-íntegra, mucosa e percutânea

outra atividade remunerada. Estudos revelaram acidente de trabalho com material biológico nos últimos proporções de $29,4 \%$ e $38 \%$ de profissionais de 2 anos de trabalho na instituição, 67 profissionais enfermagem que exerciam outro trabalho remunerado (82,7\%) afirmaram que não sofreram acidente de ${ }^{(16,23)}$. É importante salientar que a enfermagem é mal trabalho e 14 (17,3\%) responderam ter sofrido acidente. remunerada e muitos trabalhadores necessitam ter outra Uma pesquisa avaliou 1.433 profissionais de atividade para aumentar o orçamento familiar ${ }^{(23)}$. saúde, dos quais 23,9\% estiveram envolvidos com Quando questionados sobre a ocorrência de acidentes de trabalho notificados com material biológico, 
entre janeiro de 1994 a dezembro de $1999^{(6)}$. Observase que este percentual de acidentes de trabalho foi um pouco maior ao encontrado no presente estudo, mas há que se considerar a diferença no número dos sujeitos pesquisados e no intervalo de tempo investigado, em ambos os estudos.

Por outro lado, o percentual de acidentados encontrado neste estudo pode ser considerado baixo, comparativamente ao estudo realizado com profissionais de saúde de hospitais públicos do Distrito Federal em 2002 e $2003^{(12)}$, que encontrou 39,1\% de acidentados com material biológico.

Estudos realizados em outros países apresentaram percentuais variados quanto à ocorrência de acidentes de trabalho. Recentes estudos sobre lesões causadas por pérfuro-cortantes demonstraram 46\% de ocorrência de lesões no período de um ano em enfermeiras de um hospital-escola no Japão ${ }^{(20)}$, e $38 \%$ de ocorrência de pelo menos uma lesão no período de um ano em profissionais de saúde do Reino Unido ${ }^{(15)}$

Neste estudo, alguns fatores poderiam explicar o baixo percentual de acidentes com material biológico relatados quando comparados com dados da literatura.

Em estudos transversais, com coleta de dados retrospectivos, pode ocorrer o surgimento do viés de memória, onde os participantes são solicitados a lembrar de alguns eventos, e alguns indivíduos tendem a lembrar mais do fato pesquisado do que outros indivíduos $^{(22-24)}$. Outra possibilidade seria a rotina de trabalho dos profissionais, já que várias vezes eles encontravam-se atarefados no momento da entrevista, dificultando a participação na pesquisa, com conseqüente subnotificação de acidentes. Além disso, o profissional poderia sentir-se constrangido em relatar o acontecido, pela associação do acidente com a falta de atenção ou negligência no trabalho, além de reviver uma experiência traumatizante pelo sofrimento psíquico causado pelo medo da contaminação pelo vírus da imunodeficiência humana ${ }^{(3)}$.

Sobre o estado vacinal contra hepatite B, 67 profissionais $(82,7 \%)$ relataram possuir o esquema vacinal completo, e 11 deles (13,5\%) o esquema vacinal incompleto. Provavelmente este resultado deveu-se à recente e contínua campanha de vacinação para os funcionários realizada na instituição. Ainda assim, um sujeito (1,3\%) não lembra de ter sido vacinado e 2 $(2,5 \%)$ se recusaram à vacinação relatando não verem benefícios e considerarem a vacinação um procedimento de risco. Outro estudo entre profissionais acidentados de saúde encontrou um percentual maior de indivíduos não vacinados, $60 \%{ }^{(7)}$. Os profissionais da área da saúde, por estarem mais expostos a risco biológico, devem estar corretamente imunizados.

A Tabela 2 apresenta a comparação entre acidentados e não acidentados quanto ao sexo, idade, estado civil, categoria profissional, tempo de trabalho, jornada de trabalho, turno de trabalho, outra atividade remunerada e imunização contra hepatite B. A única variável que apresentou diferença significativa $(\mathrm{p}=0,04)$ entre os grupos foi o tempo de serviço na instituição, com associação entre tempo de serviço menor que 2 anos e a ocorrência de acidente.

Dados da literatura demonstram resultados quanto ao tempo de serviço e a ocorrência de acidentes, como uma recente pesquisa sobre acidentes com material pérfuro-cortante notificados, os quais foram mais freqüentes (89,5\% dos casos) entre os profissionais de saúde com 1 a 5 anos de tempo de serviço, concluindo que os acidentes ocorreram com maior freqüência nos profissionais com pouco tempo de serviço ${ }^{(9)}$.

$\mathrm{Na}$ literatura internacional algumas pesquisas analisaram o tempo de serviço como fator de risco para acidentes. Por exemplo, nos Estados Unidos, 2.730 profissionais de saúde expostos a sangue e fluidos corporais (considerando exposições percutâneas, exposições de membranas mucosas, exposições de pele e mordedura humana), foram estudados por meio de notificações de 1998 a 2002. Entre outros achados, foram considerados de mais alto risco para exposição ao sangue e fluidos corporais os trabalhadores empregados há menos de 4 anos $^{(14)}$.

Uma coorte de 24.000 trabalhadores de saúde de hospitais na França, analisando 1.929 profissionais acidentados com sangue ou fluidos com sangue (incluindo lesão percutânea, mucocutânea ou pele lesada), de 1996 a 1998, revelou que os trabalhadores com menos de 2 anos de tempo de serviço apresentaram maior risco que os mais experientes ${ }^{(10)}$. Contudo, outra pesquisa apontou um número significativamente maior de acidentes de trabalho com material biológico notificados nos profissionais com maior tempo de serviço na instituição ${ }^{(6)}$.

É importante que sejam feitas as orientações básicas do funcionamento do serviço e dos potenciais fatores de riscos a que o profissional está exposto, principalmente para os recém-contratados na instituição. Assim terão o conhecimento de como desenvolver seu trabalho e a quem se dirigir caso venham a sofrer algum acidente laboral ${ }^{(23)}$. 
Tabela 2 - Associação entre variáveis de acidentados e não acidentados com material biológico em trabalhadores de enfermagem. Paraná, Set 2004/Dez 2006

\begin{tabular}{|c|c|c|c|c|c|c|}
\hline \multirow{3}{*}{ Categorias } & \multirow{3}{*}{ Variáveis } & \multicolumn{4}{|c|}{ Acidente de trabalho } & \multirow{3}{*}{$\mathbf{P}$} \\
\hline & & \multicolumn{2}{|c|}{ SIM } & \multicolumn{2}{|c|}{ NÃO } & \\
\hline & & $\mathrm{N}=14$ & $\%$ & $\mathrm{n}=67$ & $\%$ & \\
\hline \multirow[t]{8}{*}{ Sócio - demográficas } & Sexo & & & & & \\
\hline & Feminino & 12 & 18,2 & 54 & 81,8 & \\
\hline & Masculino & 2 & 13,3 & 13 & 86,7 & $1,0^{*}$ \\
\hline & Idade & & & & & \\
\hline & Mediana & \multicolumn{2}{|c|}{31,5} & \multicolumn{2}{|c|}{33,0} & $0,64 \dagger$ \\
\hline & Estado civil & & & & & \\
\hline & Com companheiro(a) & 10 & 22,7 & 34 & 77,3 & \\
\hline & Sem companheiro(a) & 4 & 10,8 & 33 & 89,2 & $0,24 *$ \\
\hline \multirow[t]{15}{*}{ Trabalho } & Categoria Profissional & & & & & \\
\hline & Técnico de enfermagem & 12 & 20,7 & 46 & 79,3 & $0,33^{*}$ \\
\hline & Auxiliar de enfermagem & 2 & 8,7 & 21 & 91,3 & \\
\hline & Tempo de trabalho & & & & & $0,04 *$ \\
\hline & Até 2 anos & 7 & 31,8 & 15 & 68,2 & \\
\hline & $>2$ anos & 7 & 11,9 & 52 & 88,1 & \\
\hline & Jornada de trabalho $\$$ & & & & & \\
\hline & 6-8 horas & 6 & 14,6 & 35 & 85,4 & \\
\hline & 12 horas & 8 & 20,5 & 31 & 79,5 & $0,56^{*}$ \\
\hline & Turno de trabalho $\ddagger$ & & & & & \\
\hline & Diurno & 5 & 11,6 & 38 & 88,4 & \\
\hline & Noturno & 9 & 24,3 & 28 & 75,7 & $0,15^{*}$ \\
\hline & Outra atividade remunerada & & & & & \\
\hline & Sim & 8 & 17,8 & 37 & 82,2 & $1,0^{*}$ \\
\hline & Não & 6 & 16,7 & 30 & 83,3 & \\
\hline \multirow[t]{3}{*}{ Estado Vacinal } & Imunização hepatite B $\S$ & & & & & \\
\hline & Esquema vacinal completo & 12 & 17,6 & 56 & 82,4 & $1,0^{*}$ \\
\hline & $\begin{array}{l}\text { Esquema vacinal Incompleto/sem } \\
\text { Imunização }\end{array}$ & 2 & 16,7 & 10 & 83,3 & \\
\hline
\end{tabular}

*Teste Exato de Fisher, † Teste de Mann Whitney, ¥ Um funcionário folguista foi excluído, § Um não informado.

A tabela 3 apresenta as características dos 16 acidentes ocorridos. Conforme relatado anteriormente, 14 profissionais referiram ter se acidentado nos últimos 2 anos. Destes 14 profissionais, 12 referiram ter sofrido acidente de trabalho 1 vez, e 2 profissionais referiram ter sofrido 2 acidentes, totalizando 16 acidentes.

O acidente com material biológico que prevaleceu em todos os casos foi o percutâneo. Em diversas pesquisas realizadas com profissionais de saúde e profissionais de enfermagem, a ocorrência desse tipo de acidente variou de $72,1 \%$ a $90,0 \% \%^{(5-7-8,12,14)}$. Acredita-se que as atividades médica e de enfermagem favorecem a ocorrência desses acidentes, pelas práticas invasivas realizadas com freqüência por estes profissionais ${ }^{(6)}$. 
Tabela 3 - Características dos acidentes de trabalho com material biológico ocorridos com profissionais de enfermagem. Paraná, Set 2004/Dez 2006 (n=16)

\begin{tabular}{|c|c|c|c|}
\hline Categorias & Variáveis & $\mathrm{n}$ & $\%$ \\
\hline Tipo de acidente & Acidente percutâneo & 16 & 100 \\
\hline \multirow[t]{7}{*}{ Setor do acidente } & Clínica Médica e Cirúrgica & 5 & 31,3 \\
\hline & Unidade de Terapia Intensiva & 3 & 18,8 \\
\hline & Central de Materiais e Esterilização & 2 & 12,5 \\
\hline & Centro Cirúrgico & 2 & 12,5 \\
\hline & Neurologia & 2 & 12,5 \\
\hline & Maternidade & 1 & 6,2 \\
\hline & Pediatria & 1 & 6,2 \\
\hline \multirow[t]{3}{*}{ Agente causador do acidente } & Agulha* & 13 & 81,3 \\
\hline & Lâmina de bisturi & 2 & 12,5 \\
\hline & Lâmina de tricotomia & 1 & 6,2 \\
\hline \multirow[t]{7}{*}{ Circunstância do acidente } & No descarte & 6 & 37,7 \\
\hline & Reencapando agulha & 4 & 25,0 \\
\hline & Inserindo agulha no tecido & 2 & 12,5 \\
\hline & Limpando instrumental & 1 & 6,2 \\
\hline & Descarte inadequado de outro profissional & 1 & 6,2 \\
\hline & Auxiliando procedimento & 1 & 6,2 \\
\hline & Paciente se moveu & 1 & 6,2 \\
\hline \multirow[t]{2}{*}{ Material Biológico } & Sangue & 15 & 93,8 \\
\hline & Tecido epitelial & 1 & 6,2 \\
\hline \multirow[t]{2}{*}{ Região do corpo afetada } & Dedo da mão & 15 & 93,8 \\
\hline & Mão & 1 & 6,2 \\
\hline \multirow[t]{3}{*}{ Período do dia } & Noite & 9 & 56,2 \\
\hline & Manhã & 6 & 37,6 \\
\hline & Tarde & 1 & 6,2 \\
\hline
\end{tabular}

*Agulhas foram consideradas cateter venoso, agulha de sutura, agulha de injeção e agulha de raqui-anestesia.

Os ferimentos pérfuro-cortantes são um grave problema nas instituições de saúde, tanto pela freqüência como pela grave repercussão na saúde desses trabalhadores ${ }^{(5)}$. Em revisão sobre os acidentes de trabalho com material pérfuro-cortante entre trabalhadores de enfermagem, no período de 1985 e 2000, observaram-se os seguintes fatores associados à ocorrência desses acidentes: falta de conscientização, inadequada supervisão da prática, não percepção individual sobre o risco e falta de educação continuada ${ }^{(2)}$. Também existe maior dificuldade na prevenção desses acidentes, pela grande freqüência das atividades que requerem a utilização de agulhas, pela dinâmica do trabalho envolvido nestes procedimentos, pelo ritmo imposto na assistência em situações de urgência/emergência, e pelas características do processo e organização na atividade laboral dos profissionais de enfermagem ${ }^{(19)}$.

Os setores do hospital nos quais detectou-se o maior percentual de ocorrências de acidentes foram 5 (31,3\%) nas Clínicas Médica e Cirúrgica e 3 (18,8\%) no setor de UTI. Um estudo ${ }^{(9)}$ encontrou resultado semelhante, sobre acidentes ocupacionais com material pérfuro-cortante em profissionais de saúde 
onde foram mais freqüentes os acidentes nas Clínicas Cirúrgica e Médica e UTI.

Outros estudos demonstraram resultados diversos sobre esta questão, como maior percentual de acidentes de trabalho com material biológico entre profissionais da saúde nas Unidades de Emergência, Clínica Médica e $\mathrm{UTI}^{(7)}$; entre profissionais de enfermagem nas Clínicas Médica, Pediátrica e Cirúrgica $^{(18)}$; e em acidentes com sangue entre profissionais da saúde nas Unidades de Emergência e Cuidados Intensivos ${ }^{(10)}$. Considerando esses resultados, enfatiza-se a necessidade de atenção aos procedimentos que apresentam ritmo diferenciado na assistência aos pacientes como os executados em ritmo acelerado, pois podem gerar desgaste do trabalhador, expondo-os a acidentes de trabalho( ${ }^{(7)}$.

A agulha foi o agente causador do acidente de trabalho com material biológico mais freqüente aparecendo neste estudo em 13 casos (81,3\%), seguida pela lâmina de bisturi em 2 casos (12,5\%) e a lâmina de tricotomia em 1 caso (6,2\%). A agulha também foi o principal agente causador de acidentes em estudo realizado sobre acidentes de trabalho com trabalhadores de enfermagem ${ }^{(13)} \mathrm{e}$ em acidentes de trabalho entre profissionais da saúde com fluidos corpóreos $^{(8)}$ e com material perfuro-cortante ${ }^{(9)}$.

Analisando as circunstâncias em que ocorreram os acidentes, 6 (37,7\%) foram por descarte de material. Os acidentes devido ao reencape de agulha estiveram presentes em 4 casos (25\%), seguidos do ato de inserir a agulha no tecido humano em 2 acidentes (12,5\%), geralmente por punção venosa e aplicação de injeção.

Em estudo realizado com profissionais de enfermagem, os acidentes se deram principalmente durante o descarte, em $58 \%$, e, em $28 \%$ dos casos foi durante a punção venosa e em $14 \%$ ao lavar o material $^{(16)}$. Outra pesquisa sobre acidentes com material pérfuro-cortante notificados entre profissionais da saúde, os acidentes ocorreram principalmente pelo descarte do pérfuro-cortante em local impróprio $(21,6 \%)^{(9)}$. Pesquisa realizada com enfermeiras de hospitais nos Estados Unidos observou que fatores associados ao aumento de lesões percutâneas incluíram reencape de agulhas e trabalhadores temporários ${ }^{(21)}$.

O material biológico mais freqüentemente envolvido nos acidentes deste estudo foi o sangue estando presente em 15 casos (93,8\%). Encontrou-se, ainda, uma situação envolvendo tecido epitelial, durante a tricotomia facial de um paciente. Neste caso, apesar do sangue do paciente-fonte não ser visível na lâmina, o risco da presença do sangue no acidente é grande, pois o procedimento envolve um material cortante. Maior freqüência de acidentes envolvendo o sangue foi também encontrada em outras pesquisas ${ }^{(6-8,18)}$.

As regiões do corpo mais atingidas nos acidentes de trabalho foram os dedos da mão em 15 acidentes $(93,8 \%)$ e a mão em 1 acidente (6,2\%). Resultado semelhante foi encontrado na literatura, onde os acidentes com material biológico também acometeram predominantemente o dedo ${ }^{(19)}$.

Neste estudo, 9 acidentes (56,2\%) ocorreram no período da noite. Em estudo sobre a duração e qualidade do sono e os níveis de alerta durante os turnos diurno e noturno, com profissionais de enfermagem de hospital, observaram-se diferenças significativas na percepção dos estados de alerta em três momentos diferentes do turno da noite. Além disso, os níveis percebidos de alerta à noite pioravam à medida que aumentava o número de horas de trabalho, indicando que a sonolência no trabalho noturno pode prejudicar trabalhadores e pacientes a seus cuidados ${ }^{(25)}$.

Acredita-se que o ritmo de trabalho seja mais intenso durante o dia, pela maioria dos procedimentos terapêuticos serem realizados neste período, o que poderia ocasionar sobrecarga de tarefas, podendo ser significativo na ocorrência do acidente típico em hospital ${ }^{(16)}$, como observado em estudos com maior freqüência de acidentes no período da manhã ${ }^{(8,18)}$.

Sobre a utilização de EPI (Tabela 4), 12 (75\%) participantes da pesquisa não utilizavam EPI no momento do acidente. Percentual elevado de não uso de EPI (60\%) no momento do acidente também foi encontrado em outro estudo sobre acidente de trabalho em UTI com profissionais de enfermagem, onde também se observou que os trabalhadores avaliam o procedimento e julgam a necessidade ou não de uso do EPI, não valorizando a real importância do seu uso para a prevenção dos acidentes laborais ${ }^{(13)}$.

A utilização das precauções-padrão como barreira ajuda no controle da transmissão de patógenos nas instituições hospitalares. A adesão ao uso de equipamentos de proteção coletiva (EPC), quando necessário, e utilização de EPI é essencial para a proteção do profissional. Contudo, tais precauções nem sempre são obedecidas resultando em um alto índice de acidentes de trabalho com material biológico pelos profissionais de saúde ${ }^{(19)}$. Muitos profissionais de saúde admitem que se preocupam com os acidentes, porém uma parte reduzida deles utilizam EPI's ${ }^{(3)}$.

Os EPI's devem ser utilizados na manipulação 
de artigos médico-hospitalares e no atendimento a definido ou suspeito de doença infecto-contagiosa, todos os pacientes, independente do diagnóstico como HIV/AIDS e hepatites B e $\mathrm{C}^{(11)}$.

Tabela 4 - Uso de Equipamento de Proteção Individual e condutas realizadas após os acidentes de trabalho com material biológico ocorridos com profissionais de enfermagem. Paraná, set 2004/Dez 2006 (n=16)

\begin{tabular}{|c|c|c|c|}
\hline Categorias & Variáveis & $\mathbf{n}$ & $\%$ \\
\hline \multirow[t]{7}{*}{ EPI } & Utilização de EPI & & \\
\hline & Não & 12 & 75,0 \\
\hline & Sim & 4 & 25,0 \\
\hline & EPI utilizado $(n=4)$ & & \\
\hline & Luva de procedimento + máscara + gorro + avental/capote de tecido & 2 & 50,0 \\
\hline & Luva de procedimento + avental/capote impermeável & 1 & 25,0 \\
\hline & Luva de procedimento & 1 & 25,0 \\
\hline \multirow{26}{*}{$\begin{array}{l}\text { Procedimentos pós- } \\
\text { acidentes }\end{array}$} & Conduta após o acidente & & \\
\hline & Água e sabão + espremeu + álcool 70\% & 6 & 37,7 \\
\hline & Água e sabão + espremeu + álcool 70\% + antisséptico & 2 & 12,5 \\
\hline & Água e sabão + álcool 70\% & 2 & 12,5 \\
\hline & Água e sabão + espremeu & 2 & 12,5 \\
\hline & Água e sabão + espremeu + álcool 70\% + antisséptico + curativo & 1 & 6,2 \\
\hline & Água e sabão + espremeu + álcool 70\% + curativo & 1 & 6,2 \\
\hline & Álcool 70\% + antisséptico & 1 & 6,2 \\
\hline & Água e sabão & 1 & 6,2 \\
\hline & Comunicação do acidente & & \\
\hline & Enfermeira $+\mathrm{CAT}^{*}+\mathrm{SCIH} \dagger$ & 8 & 50,2 \\
\hline & Enfermeira + SCIH & 2 & 12,5 \\
\hline & Livro de registro da unidade $+\mathrm{SAE} \ddagger$ & 2 & 12,5 \\
\hline & Enfermeira $+\mathrm{CAT}+\mathrm{SCIH}+\mathrm{SAE}$ & 1 & 6,2 \\
\hline & Enfermeira + livro de registro da unidade & 1 & 6,2 \\
\hline & Enfermeira & 1 & 6,2 \\
\hline & Não comunicado & 1 & 6,2 \\
\hline & Realização do exame de sangue do paciente-fonte & & \\
\hline & Sim & 10 & 62,5 \\
\hline & Não & 6 & 37,5 \\
\hline & Realização do exame de sangue do profissional & & \\
\hline & Sim & 13 & 81,3 \\
\hline & Não & 3 & 18,7 \\
\hline & Realização de profilaxia medicamentosa & & \\
\hline & Não & 16 & 100 \\
\hline & Sim & 0 & 0 \\
\hline
\end{tabular}

* CAT: Comunicação de Acidente de Trabalho, † SCIH: Serviço de Controle de Infecção Hospitalar, ł SAE: Serviço de Atendimento Especializado 
A utilização do jaleco não foi considerada nesta pesquisa, pois faz parte do uniforme de trabalho do profissional, com exceção de quem trabalha no Centro Cirúrgico, UTI e Pronto-socorro, que possui uniforme fornecido pelo hospital. Dos 16 acidentes de trabalho com material biológico que foram estudados, em apenas 4 deles os profissionais utilizavam EPI. Nesses 4 acidentes, os profissionais relataram estar usando luva de procedimento, sendo que em 3 deles utilizavam também outros EPIs. Em pesquisa realizada, observouse que o EPI mais utilizado no momento do acidente foi a luva de procedimento, em $74,3 \%$ dos casos, embora uma parte dos profissionais usassem luvas apenas em locais de risco máximo (emergência, centro cirúrgico, hemodiálise etc.), o que não é apropriado ${ }^{(6)}$.

As luvas são indicadas sempre que houver possibilidade de contato com sangue, secreções e excreções, com mucosas ou com áreas de pele nãoíntegra. Mesmo não existindo um benefício cientificamente comprovado de redução dos riscos de transmissão de patógenos sangüíneos, o uso de duas luvas reduz de forma significativa a contaminação das mãos com sangue e tem sido recomendado em cirurgias com alto risco de exposições (por exemplo, em cirurgias obstétricas, ortopédicas ou torácicas) ${ }^{(11)}$.

As normas de precauções devem ser utilizadas na assistência a todos os pacientes na manipulação de sangue, secreções e excreções e contato com mucosa e pele não-íntegra. Essas medidas incluem a utilização de EPI's e os cuidados específicos recomendados para manipulação e descarte de materiais pérfuro-cortantes contaminados por materiais orgânicos. Os EPIs recomendados, conforme as atividades desenvolvidas são luvas, máscara, gorro, óculos de proteção, capotes (aventais) e bota ${ }^{(3)}$.

Ocorre certa relutância do trabalhador em mudar sua rotina no que se refere às técnicas realizadas, a não-disponibilidade do material para proteção, e a falta de apoio do setor administrativo ${ }^{(4)}$. Além disso, muitos EPIs são desconfortáveis e podem atrapalhar a prática profissional pela restrição dos movimentos ${ }^{(11)}$.

Quanto à conduta no local da lesão após o acidente, observou-se que todos os profissionais realizaram alguma conduta, sendo que 6 profissionais (37,7\%) utilizaram água, sabão, espremeram e aplicaram álcool 70\%. Estudos demonstraram que a quase totalidade dos profissionais realizou alguma conduta no local da lesão após o acidente ${ }^{(7,18)}$. Apesar da prática rotineiramente recomendada de lavar o local da lesão com água e sabão, não há estudos que comprovem a eficácia deste procedimento na redução do risco à infecção ${ }^{(7)}$.

Em relação às medidas de comunicação do acidente de trabalho, com exceção de um que não foi comunicado, todos os outros tiveram algum tipo de comunicação, sendo que em 8 casos (50,2\%) houve comunicação sobre o acidente à Enfermeira da unidade, abertura de CAT (Comunicação de Acidente de Trabalho) e encaminhamento para a SCIH (Serviço de Controle de Infecção Hospitalar). Contudo, observou-se a não padronização das comunicações e acompanhamento dos acidentados estudados.

Para que os benefícios dos acidentados sejam garantidos, os hospitais devem ter registro desses acidentes. Portanto, é importante o estabelecimento de rotinas relacionadas às medidas de precauções imediatas, às orientações e à disponibilidade da quimioprofilaxia em casos de acidentados com material biológico de pacientes soropositivos para o HIV ou desconhecidos $^{(12)}$.

Quanto à realização de exames de sangue para sorologias de HIV, HBV e HCV, em 10 casos (62,5\%) foi coletado sangue do paciente-fonte e em 13 casos (81,3\%) foi coletado sangue dos profissionais acidentados. Nenhum dos sujeitos da pesquisa recebeu profilaxia medicamentosa. Outros autores encontraram $96,3 \%$ de registros da sorologia do profissional e o conhecimento sobre a sorologia do paciente estava registrado em apenas $74,08 \%$ das fichas preenchidas sobre acidentes com material biológico em trabalhadores de enfermagem ${ }^{(19)}$.

Segundo protocolo do Ministério da Saúde, o profissional de saúde acidentado com material biológico deve comunicar à chefia da unidade onde trabalha sobre o acidente, deve ser preenchida a CAT, investigada a gravidade do acidente e sobre o fluido corpóreo de risco, e realizados os exames necessários. O acidentado deve ser encaminhado o mais rápido possível para avaliação e conduta, seguido de acompanhamento clínico, sorológico e psicológico, quando necessário ${ }^{(26)}$.

\section{CONCLUSÃO}

Este estudo permitiu caracterizar o perfil dos técnicos e auxiliares de enfermagem de um hospital geral quanto aos aspectos: sociodemográficos, do trabalho, estado vacinal, acidentes de trabalho ocorridos com material biológico e analisar as condutas realizadas após o acidente, fornecendo informações 
que podem direcionar medidas específicas de intervenções na organização do trabalho, visando a prevenção de acidentes.

Pelos resultados obtidos, evidenciou-se a importância da orientação sobre o funcionamento do hospital e os riscos a que estão expostos os profissionais de enfermagem, com especial atenção aos com menos tempo de serviço.

O não uso de EPI's no momento do acidente teve um elevado índice, e observou-se falhas na comunicação do acidente. Portanto, enfatiza-se a importância da educação permanente para os profissionais sobre utilização de EPIs, e orientações adequadas acerca do protocolo de medidas administrativas sobre a comunicação de acidente de trabalho com material biológico.

Estudos desta natureza servem como fonte de informações para que profissionais de saúde, empregadores e administradores atentem com mais seriedade para o desenvolvimento de estratégias para redução da exposição aos riscos a que estão expostos durante o trabalho, como a implantação de medidas preventivas e de conscientização, bem como a efetivação de condutas corretas a serem tomadas após um acidente de trabalho com material biológico.

Sugerem-se novas pesquisas sobre o tema, com coleta de dados de maneira prospectiva, incluindo diferentes instituições para comparação e outras abordagens metodológicas, para melhor entendimento das questões relacionadas com acidente de trabalho com exposição à material biológico em profissionais de enfermagem.

\section{REFERÊNCIAS}

1. Takayanagui AMM, Mendes MD, Dias L. Biossegurança, enfermagem e os resíduos de serviços de saúde. Rev Bras Saúde Ocup. 1993;21(80):23-7.

2. Marziale MHP, Rodrigues CM. A produção científica sobre os acidentes de trabalho com material pérfurocortante entre trabalhadores de enfermagem. Rev Latino-Am Enferm. 2002;10(4):571-7.

3. Brandão Júnior PS. Biossegurança e AIDS: as dimensões psicossociais do acidente com material biológico no trabalho em hospital [dissertação]. Rio de Janeiro (RJ): Fundação Oswaldo Cruz, Escola Nacional de Saúde Pública; 2000.

4. Queiroz MCB. Biossegurança. In: Albuquerque CP, Oliveira AC, Rocha LCM. Infecções hospitalares: abordagem, prevenção e controle. Rio de Janeiro: MEDSI; 1998. p.183-96.

5. Sarquis LMM, Felli VEA, Miranda FMDA, Guimarães HV, Oliveira GP. A adesão ao protocolo de monitoramento dos trabalhadores de saúde após exposição a fluidos biológicos: uma problemática vivenciada em um ambulatório de saúde do trabalhador no Paraná. Cogitare Enferm. 2005 Mai/Ago;10(2):47-53.

6. Ciorlia LAS, Zanetta DMT. Significado epidemiológico dos acidentes de trabalho com material biológico: hepatite B e C em profissionais da saúde. Rev Bras Med Trab. 2004;2(3):191-9.

7. Balsamo AC, Felli VEA. Estudos sobre os acidentes de trabalho com exposição aos líquidos corporais humanos em trabalhadores da saúde de um hospital universitário. Rev Latino-Am Enferm. 2006;14(3):346-53.

8. Almeida CAF, Benatti MCC. Exposições ocupacionais por fluidos corpóreos entre trabalhadores da saúde e sua adesão à quimioprofilaxia. Rev Esc Enferm USP. 2007;41(1):120-6.

9. Moura JP, Gir E, Canini SRMS. Acidentes ocupacionais com material pérfuro-cortante em um Hospital Regional de Minas Gerais, Brasil. Cienc Enferm. 2006;12 (1):29-37.

10. Denis M-A, Ecochard R, Bernadet A, Forissier M-F, Porst J-M, Robert O, et al. Risk of occupational blood exposure in a cohort of 24.000 hospital healthcare workers: position and environment analysis over three years. J Occup Environ Med. 2003;45(3):283-8.

11. Ministério da Saúde (BR). Manual de exposição ocupacional: recomendações para atendimento e acompanhamento de exposição ocupacional a material biológico HIV, Hepatites B e C. Normas do Programa Nacional DST/AIDS. Brasília; 2004.

12. Caixeta RB, Barbosa-Branco A. Acidente de trabalho, com material biológico, em profissionais de saúde de hospitais públicos do Distrito Federal, Brasil, 2002/2003. Cad Saúde Públ. 2005;21(3):737-46.

13. Nishide VM, Benatti MCC, Alexandre NMC. Ocorrência de acidente de trabalho em uma Unidade de Terapia Intensiva. Rev Latino-Am Enferm. 2004;12(2):204-11.

14. Dement JM, Epling C, Ostbye T, Pompeii LA, Hunt DL. Blood and body fluid exposure risks among health care workers: results from the Duke Health and Safety Surveillance System. Am J Ind Med. 2004;46:637-48.

15. Elmiyeh B, Whitaker IS, James MJ, Chahal CAA, Galea 
A, Alshafi K. Needle-stick injuries in the National Health Service: a culture of silence. J R Soc Med.2004;97:326-7.

16. Braga D. Acidente de trabalho com material biológico em trabalhadores da equipe de enfermagem do Centro de Pesquisas Hospital Evandro Chagas [dissertação]. Rio de Janeiro (RJ): Fundação Oswaldo Cruz, Escola Nacional de Saúde Pública; 2000.

17. Sarquis LMM, Felli VEA. O uso dos equipamentos de proteção individual entre os trabalhadores de enfermagem acidentados com instrumentos pérfurocortantes. Rev Bras Enferm. 2000;53(4):564-73.

18. Nhamba LA. Acidentes ocupacionais com material biológico entre profissionais de enfermagem em um hospital de Angola, 2004 [dissertação]. Ribeirão Preto (SP): Escola de Enfermagem de Ribeirão Preto, Universidade de São Paulo; 2004.

19. Sêcco IAO, Leroux AMR, Santos CF, Robazzi MLCC. Epidemiologia dos acidentes de trabalho com material biológico na equipe de enfermagem de hospital público do Paraná, UNOPAR. Cient Ciênc Biol Saúde. 2002;4(2):37-43.

20. Smith DR, Mihashi M, Adachi Y, Nakashima Y, Ishitake T. Epidemiology of needlestick and sharps injuries among nurses in a Japanese teaching hospital. J Hosp Infect. 2006;64:44-9.

21. Aiken LH, Sloane DM, Klocinski JL. Hospital nurses' occupational exposure to blood: prospective, retrospective, and institutional reports. Am J Public Health. 1997;87(1):103-7.

22. Pereira MG. Epidemiologia: teoria e prática. Rio de Janeiro: Guanabara Koogan S.A; 1995. p.596.

23. Rezende MP. Agravos à saúde de auxiliares de enfermagem resultantes da exposição ocupacional aos riscos físicos, 2003 [dissertação]. Ribeirão Preto (SP): Escola de Enfermagem de Ribeirão Preto, Universidade de São Paulo; 2003.

24. Dawson B, Trapp RG. Tradutores Vorsatz CM, Cosendey CHA, Valejo MA. Interpretação da literatura médica. In: Dawson B, Trapp RG . Bioestatística Básica e Clínica. $3^{\mathrm{a}}$ ed. Rio de Janeiro: McGraw-Hill Interamericana do Brasil Ltda; 2003. p.271-96.

25. Fischer FM, Teixeira LR, Borges FNS, Gonçalves MBL, Ferreira RM. Percepção de sono: duração, qualidade e alerta em profissionais da área de enfermagem. Cad Saúde Públ. 2002;18(5):1261-9.
26. Secretaria de Estado da Saúde [Homepage na internet]. Curitiba: Secretaria de Estado da Saúde; Centro Estadual de Saúde do Trabalhador. Saúde Paraná; [Acesso em 2007 julho 08]. Protocolo de atendimento de acidentes de trabalho com exposição a material biológico (hepatites e HIV). Disponível em: http:// www.saude.pr.gov.br/saudedotrabalhador/index.html 